Journal of Mathematics and Statistics 2 (1): 360-362, 2006

ISSN 1549-3652

(C) 2006 Science Publications

\title{
Some Stability Results on Krasnolslseskij and Ishikawa Fixed Point Iteration Procedures
}

\author{
M. O. Olatinwo, O. O. Owojori and C. O. Imoru \\ Department of Mathematics, Obafemi Awolowo University, Ile-Ife, Nigeria
}

\begin{abstract}
In this study, we establish some stability results for the Krasnoselskij and the Ishikawa iteration procedures. We employ the same method as in Berinde ${ }^{[1]}$, but using a more general contractive definition than those of Berinde ${ }^{[1]}$, Rhoades $^{[2]}$, Harder and Hicks ${ }^{[3]}$ and Osilike ${ }^{[4,5]}$.
\end{abstract}

Key words: Krasnolslseskij, Ishikawa, iteration procedures, contractive definitions

\section{INTRODUCTION}

Let $(\mathrm{E}, \mathrm{d})$ be a complete metric space and $\mathrm{T}: \mathrm{E} \rightarrow \mathrm{E}$ a selfmap of $E$. Let $F(T)=\left\{p \in E \mid T_{p=p}\right\}$ denote the set of fixed points of $\mathrm{T}$. Let $\left\{\mathrm{x}_{\mathrm{n}}\right\}_{n=o}^{\infty}$ be the sequence generated by an iteration procedure

$\mathrm{x}_{\mathrm{n}+1}=f\left(\mathrm{~T}, \mathrm{x}_{\mathrm{n}}\right), \mathrm{n}=0,1,2, \ldots$

Where, $\mathrm{x}_{0} \in \mathrm{E}$ is the initial approximation and $f$ is some function. Suppose that $\left\{\mathrm{x}_{\mathrm{n}}\right\}_{n=o}^{\infty}$ converges to a fixed point $\mathrm{p}$ of $\mathrm{T}$.

Let $\left\{\mathrm{y}_{\mathrm{n}}\right\}_{n=o}^{\infty} \subset \mathrm{E}$ and set $\in_{\mathrm{n}}=\mathrm{d}\left(\mathrm{y}_{\mathrm{n}+1}, f\left(\mathrm{~T}, \mathrm{y}_{\mathrm{n}}\right)\right), \mathrm{n}=$ $0,1,2, \ldots$ Then, the iteration procedure (I) is said to be $\mathrm{T}$-stable with respect to $\mathrm{T}$, if and only if, $\lim _{n \rightarrow \infty} \in_{n}=0$ implies $\lim _{n \rightarrow \infty} y_{n}=p$

Harder and Hicks ${ }^{[3]}$ employed the concept above in proving several stability results under various contractive definitions. Rhoades ${ }^{[2,6]}$ extended the results of Harder and Hicks ${ }^{[3]}$ to other classes of contractive mappings. Specifically, Rhoades ${ }^{[2]}$ extended the results of Harder and Hicks ${ }^{[3]}$ to the following contractive definition: there exists a constant $\mathrm{c}, 0 \leq \mathrm{c}<1$ such that, for each $\mathrm{x}, \mathrm{y} \in \mathrm{E}$,

$$
\begin{aligned}
\left\|\mathrm{T}_{\mathrm{x}}-\mathrm{T}_{\mathrm{y}}\right\| & \leq \mathrm{c} \max \left\{\|\mathrm{x}-\mathrm{y}\|, \frac{1}{2}(\|\mathrm{x}-\mathrm{Tx}\|\right. \\
& \left.\left.+\left\|\mathrm{y}-\mathrm{T}_{\mathrm{y}}\right\|\right),\left\|\mathrm{x}-\mathrm{T}_{\mathrm{y}}\right\|,\left\|\mathrm{y}-\mathrm{T}_{\mathrm{x}}\right\|\right\} .
\end{aligned}
$$

Using (2), Rhoades ${ }^{[2]}$ established several stability results which are generalizations of the results of Harder and Hicks ${ }^{[3]}$. It was shown in Rhoades ${ }^{[2]}$ that

$$
\mathrm{d}(\mathrm{Tx}, \mathrm{Ty}) \leq \frac{c}{1-c} \mathrm{~d}(\mathrm{x}, \mathrm{Tx})+\mathrm{cd}(\mathrm{x}, \mathrm{y})
$$

Osilike $^{[4]}$ extended the results of Rhoades ${ }^{[2]}$ to the following contractive definition: there exist constants $\mathrm{L}$ $\geq 0, a \in[0,1)$ such that, for each $\mathrm{x}, \mathrm{y} \in \mathrm{E}$

$\mathrm{d}(\mathrm{Tx}, \mathrm{Ty}) \leq \operatorname{Ld}(\mathrm{x}, \mathrm{Tx})+\operatorname{ad}(\mathrm{x}, \mathrm{y})$.

Osilike $^{[4]}$ proved several stability results using (4). Most of the results of Osilike ${ }^{[4]}$ are generalizations of the results of Rhoades ${ }^{[2]}$ which are themselves generalizations of the results of Harder and Hicks ${ }^{[3]}$. Berinde $^{[1]}$ using a different method, proved the same results as Harder and Hicks ${ }^{[3]}$ for the same iteration procedures, using the contractive definition (4) above.

In this study, we present some stability results for Krasnoselskij and Ishikawa iteration processes using a more general contractive definition than those of Harder and Hicks ${ }^{[3]}$, Osilike ${ }^{[4]}$, Rhoades ${ }^{[2,6]}$ and Berinde $^{[1]}$. We shall however employ the method of Berinde $^{[1]}$ in our proofs.

Preliminaries: Let $\left\{\mathrm{x}_{\mathrm{n}}\right\}_{n=o}^{\infty}$ be the sequence generated by the iteration procedure (1). Then, the Krasnoselskij iteration procedure is obtained from (1) when $f\left(\mathrm{~T}, \mathrm{x}_{\mathrm{n}}\right)=$ $\frac{1}{2}\left(x_{n}+T_{n}\right), n \geq 0$, while the Ishikawa iteration process is obtained from (1) when $f\left(T, x_{n}\right)=\left(1-\alpha_{n}\right) x_{n}+\alpha_{n} T z_{n}$,

$$
z_{n}=\left(1-\beta_{n}\right) x_{n}+\beta_{n} T x_{n}, n \geq 0 .
$$

We shall employ the following contractive definition: there exist a constant $b \in[0,1)$ and a monotone increasing function $\varphi: \mathfrak{R}_{+} \rightarrow \mathfrak{R}_{+}$with $\varphi(0)=$ 0 , such that, for each $\mathrm{x}, \mathrm{y} \in \mathrm{E}$,

$\|\mathrm{Tx}-\mathrm{Ty}\| \leq \varphi\|\mathrm{x}-\mathrm{Tx}\|+\mathrm{b}\|\mathrm{x}-\mathrm{y}\|$. 
The contractive definition (5) is more general in the following sense: If $\varphi(u)=L u, L \geq 0$ in (5), then we obtain the contractive mapping of Osilike ${ }^{[4]}$. If $\varphi(u)$ $=\frac{c}{1-c} \mathrm{u}$ in (5), then we have the contractive mapping of Rhoades $^{[2]}$. Also, if $\mathrm{L}=2 \delta$ and $\mathrm{a}=\delta \mathrm{in}^{[3]}$ where $\delta=$ $\max \left\{\alpha, \frac{\beta}{1-\beta}, \frac{\gamma}{1-\gamma}\right\}, 0 \leq \alpha<1,0 \leq \beta<0.5,0 \leq \Upsilon \leq 0.5$, then we obtain the Zamfirescu's contraction in Harder and Hicks ${ }^{[3]}$ and Berinde ${ }^{[1]}$. When $\varphi(\mathrm{u})=0$, then (5) reduces to

$\|\mathrm{Tx}-\mathrm{Ty}\| \leq \mathrm{b}\|\mathrm{x}-\mathrm{y}\|, \mathrm{b} \in[0,1)$,

which is a contractive definition in Harder and Hicks ${ }^{[3]}$, Berinde $^{[1]}$.

In the sequel, we shall require the following Lemma due to Berinde ${ }^{[1]}$.

Lemma 1 (Berinde ${ }^{[1]}$ ): If $\delta$ is a real number such that 0 $\leq \delta<1$, and $\left\{\epsilon_{\mathrm{n}}\right\}_{n=o}^{\infty}$ is a sequence of positive numbers such that $\lim _{n \rightarrow \infty} \epsilon_{n}=0$, then for any sequence of positive numbers $\left\{\mathrm{u}_{\mathrm{n}}\right\}_{n=o}^{\infty}$ satisfying $\mathrm{u}_{\mathrm{n}+1} \leq \delta \mathrm{u}_{\mathrm{n}}+\epsilon_{\mathrm{n}}, \mathrm{n}=0,1 \ldots$ we have $\lim _{n \rightarrow \infty} u_{n}=0$

\section{MAIN RESULTS}

We first establish a stability result for the Krasnolseskij iteration procedure as follows.

Theorem 1: Let $\left\{\mathrm{y}_{\mathrm{n}}\right\}_{n=o}^{\infty} \subset \mathrm{E}$ and $\epsilon_{\mathrm{n}}=\| \mathrm{y}_{\mathrm{n}+1}-\frac{1}{2}\left(\mathrm{y}_{\mathrm{n}}+\right.$ $\left.\mathrm{Ty}_{\mathrm{n}}\right) \|$. Let $(\mathrm{E},\|\cdot\|)$ be a normed linear space and $\mathrm{T}$ : $\mathrm{E}$ $\rightarrow$ E a selfmap of E satisfying (5). Suppose $\mathrm{T}$ has a fixed point $p$. For arbitrary $\mathrm{x}_{0}, \in \mathrm{E}$, define sequence $\left\{\mathrm{x}_{\mathrm{n}}\right\}_{n=o}^{\infty}$ iteratively by; $\mathrm{x}_{\mathrm{n}+1}=f\left(\mathrm{~T}, \mathrm{x}_{\mathrm{n}}\right)=\frac{1}{2}\left(\mathrm{x}_{\mathrm{n}}+\mathrm{Tx}_{\mathrm{n}}\right), \mathrm{n} \geq 0$.

Let $\varphi: \Re_{+} \rightarrow \Re_{+}$be monotone increasing with $\varphi(0)=0$. Then, the Krasnolseskij iteration process is T-stable.

Proof: Let $\lim _{n \rightarrow \infty} \in_{n}=0$. We shall establish that $\lim _{n \rightarrow \infty} y_{n}=p$. Using (5) and the triangle inequality:

$$
\begin{aligned}
\left\|y_{n+1}-p\right\| & \leq\left\|y_{n+1}-\frac{1}{2}\left(y_{n}+T y_{n}\right)\right\|+\left\|\frac{1}{2}\left(T y_{n}-p\right)\right\| \\
& =\epsilon_{n}+\frac{1}{2}\left\|\left(y_{n}-p\right)+\left(T y_{n}-p\right)\right\| \\
& \leq \frac{1}{2}\left\{\left\|y_{n}-p\right\|+\left\|T y_{n}-p\right\|\right\}+\epsilon_{n}
\end{aligned}
$$

$$
\begin{aligned}
& =\frac{1}{2}\left\{\left\|y_{n}-p\right\|+\left\|p-T y_{n}\right\|\right\}+\epsilon_{n} \\
& =\frac{1}{2}\left\{\left\|y_{n}-p\right\|+\left\|T p-T y_{n}\right\|\right\}+\epsilon_{n} \\
& \leq \frac{1}{2}\left\{\left\|y_{n}-p\right\|+\varphi(\|p-T p\|)+b\left\|p-y_{n}\right\|\right\}+\epsilon_{n} \\
& \left(\frac{1+b}{2}\right)\left\|y_{n}-p\right\|+\epsilon_{n}
\end{aligned}
$$

Since $0 \leq\left(\frac{1+b}{2}\right)<1$, then by Lemma 1 , (8) yields $\lim _{n \rightarrow \infty}\left\|\mathrm{y}_{\mathrm{n}}-\mathrm{p}\right\|=0$.

This implies that $\lim _{n \rightarrow \infty} y_{n}=p$

Conversely, suppose $\lim _{n \rightarrow \infty} y_{n}=p$. Then, from our hypothesis

$$
\begin{aligned}
\epsilon_{\mathrm{n}} & =\left\|\mathrm{y}_{\mathrm{n}+1}-\frac{1}{2}\left(\mathrm{y}_{\mathrm{n}}+T \mathrm{y}_{\mathrm{n}}\right)\right\| \\
& \leq\left\|\mathrm{y}_{\mathrm{n}+1}-\mathrm{p}\right\|+\left\|\mathrm{p}-\frac{1}{2}\left(\mathrm{y}_{\mathrm{n}}+T \mathrm{y}_{\mathrm{n}}\right)\right\| \\
& =\left\|\mathrm{y}_{\mathrm{n}+1}-\mathrm{p}\right\|+\left\|\frac{1}{2}\left(\mathrm{p}-\mathrm{y}_{\mathrm{n}}+\mathrm{p}-\mathrm{Ty}_{\mathrm{n}}\right)\right\| \\
& \left.\leq\left\|\mathrm{y}_{\mathrm{n}+1}-\mathrm{p}\right\|+\frac{1}{2}\left\|\mathrm{y}_{\mathrm{n}}-\mathrm{p}\right\|+\frac{1}{2} \| \mathrm{p}-T \mathrm{y}_{\mathrm{n}}\right) \| \\
& \left.=\left\|\mathrm{y}_{\mathrm{n}+1}-\mathrm{p}\right\|+\frac{1}{2}\left\|\mathrm{y}_{\mathrm{n}}-\mathrm{p}\right\|+\frac{1}{2} \| \mathrm{Tp}-\mathrm{Ty_{n }}\right) \| \\
\leq & \left\|\mathrm{y}_{\mathrm{n}+1}-\mathrm{p}\right\|+\frac{1}{2}\left[\varphi(\|\mathrm{p}-\mathrm{Tp}\|)+\mathrm{b}\left\|\mathrm{p}-\mathrm{y}_{\mathrm{n}}\right\|\right]+\frac{1}{2}\left\|\mathrm{y}_{\mathrm{n}}-\mathrm{p}\right\| \\
= & \left\|\mathrm{y}_{\mathrm{n}+1}-\mathrm{p}\right\|+\left(\frac{1+b}{2}\right)\left\|\mathrm{y}_{\mathrm{n}}-\mathrm{p}\right\| \rightarrow 0 \text { as } \mathrm{n} \rightarrow \infty .
\end{aligned}
$$

This completes the proof.

Remark 1: By similar argument as above, it is easy to establish a more general case of Theorem 1, which is stated as follows:

Theorem 2: Let $\left\{\mathrm{y}_{\mathrm{n}}\right\}_{n=o}^{\infty} \subset \mathrm{E}$ and $\in_{\mathrm{n}}=\| \mathrm{y}_{\mathrm{n}+1}-(1-\mathrm{a}) \mathrm{y}_{\mathrm{n}}$ - $\operatorname{aTy}_{n} \|, n \geq 0$. Let $(E,\|\|$.$) be a normed linear space$ and $\mathrm{T}: \mathrm{E} \rightarrow \mathrm{E}$ a selfmap of $\mathrm{E}$ satisfying (5). Suppose $\mathrm{T}$ has a fixed point $\mathrm{p}$. For arbitrary $\mathrm{x}_{0}, \in \mathrm{E}$, define sequence $\left\{\mathrm{x}_{\mathrm{n}}\right\}_{n=o}^{\infty}$ iteratively by: $\mathrm{x}_{\mathrm{n}+1}=f\left(\mathrm{~T}, \mathrm{x}_{\mathrm{n}}\right)=(1-$ a) $\left.x_{n}+a T x_{n}\right), n \geq 0, a \in[0,1]$. Let $\varphi: \Re_{+} \rightarrow \Re_{+}$be monotone increasing and $\varphi(0)=0$. Then, the Schaefer's iteration process(or the Krasnolseskij iteration in the general form) is T-stable.

Remark 2: Specifically, if a $=1$ in Theorem 2, we obtain the stability result for the Picard iteration process, (Imoru and Olatinwu ${ }^{[7]}$ and if $a=\frac{1}{2}$ in Theorem above, we obtain Theorem 1 . 
We now establish stability result for the Ishikawa iteration process.

Theorem 3: Let $\left\{\mathrm{y}_{\mathrm{n}}\right\}_{n=o}^{\infty} \subset \mathrm{E}$ and define $\mathrm{s}_{\mathrm{n}}=\left(1-\beta_{\mathrm{n}}\right) \mathrm{y}_{\mathrm{n}}$ $+\beta_{\mathrm{n}} T y_{\mathrm{n}}, \mathrm{n} \geq 0$, let $\in_{\mathrm{n}}=\left\|\mathrm{y}_{\mathrm{n}+1}-(1-\alpha) \mathrm{y}_{\mathrm{n}}-\alpha \mathrm{T} \mathrm{s}_{\mathrm{n}}\right\|$. Let $(\mathrm{E}$, $\|\|$.$) be a normed linear space and \mathrm{T}: \mathrm{E} \rightarrow \mathrm{E}$ a selfmap of E satisfying (5). Suppose T has a fixed point $p$ and $\varphi$ : $\mathfrak{R}_{+} \rightarrow \mathfrak{R}_{+}$is monotone increasing and $\varphi(0)=0$. For arbitrary $\mathrm{x}_{0}, \in \mathrm{E}$, define sequence $\left\{\mathrm{x}_{\mathrm{n}}\right\}_{n=o}^{\infty}$ iteratively by:

$$
\left.\begin{array}{l}
\mathrm{x}_{\mathrm{n}+1}=\left(1-\alpha_{\mathrm{n}}\right) \mathrm{x}_{\mathrm{n}}+\alpha_{\mathrm{n}} \mathrm{Tz}_{\mathrm{n}} \\
\mathrm{z}_{\mathrm{n}}=\left(1-\beta_{\mathrm{n}}\right) \mathrm{x}_{\mathrm{n}}+\beta_{\mathrm{n}} \mathrm{Tx}_{\mathrm{n}}
\end{array}\right\} \mathrm{n} \geq 0
$$

Where, $\left\{\alpha_{n}\right\}_{n=o}^{\infty}$ and $\left\{\beta_{n}\right\}_{n=o}^{\infty}$ are real sequences satisfying:

(i) $\alpha_{\mathrm{o}}=1$

(ii) $0 \leq \alpha_{\mathrm{n}}, \beta_{\mathrm{n}} \leq 1, \mathrm{n} \geq 0$ and

(iii) $0 \leq\left(1-\alpha_{n}+\alpha_{n} b-\alpha_{n} \beta_{n} b+\alpha_{n} \beta_{n} b^{2}\right) \leq\left(1-\alpha_{n}+\alpha_{n} b\right)<1$.

Then, the Ishikawa iteration process is T-stable.

\section{Proof:}

$$
\begin{aligned}
& \left\|y_{n+1}-p\right\| \leq\left\|y_{n+1}-\left(1-\alpha_{n}\right) y_{n}-\alpha_{n} T s_{n}\right\| \\
& \left.\quad+\| 1-\alpha_{n}\right) y_{n}-\alpha_{n} T s_{n}-p \| \\
& =\epsilon_{n}+\left\|\left(1-\alpha_{n}\right) y_{n}+\alpha_{n} T_{n}-\left[\left(1-\alpha_{n}\right)+\alpha_{n}\right] p\right\| \\
& =\left\|\left(1-\alpha_{n}\right)\left(y_{n}-p\right)+\alpha_{n}\left(\operatorname{Ts}_{n}-p\right)\right\|+\epsilon_{n} \\
& \leq\left(1-\alpha_{n}\right)\left\|y_{n}-p\right\|+\alpha_{n}\left\|T s_{n}-p\right\|+\epsilon_{n} \\
& =\left(1-\alpha_{n}\right)\left\|y_{n}-p\right\|+\alpha_{n}\left\|p-T s_{n}\right\|+\epsilon_{n} \\
& =\left(1-\alpha_{n}\right)\left\|y_{n}-p\right\|+\alpha_{n}\left\|T p-T s_{n}\right\|+\epsilon_{n}
\end{aligned}
$$

Since T satisfies (5), we have:

$\left\|\mathrm{y}_{\mathrm{n}+1}-\mathrm{p}\right\| \leq\left(1-\alpha_{\mathrm{n}}\right)\left\|\mathrm{y}_{\mathrm{n}}-\mathrm{p}\right\|+\alpha_{\mathrm{n}}\left[\varphi(\mathrm{p}-\mathrm{Tp} \|)+\mathrm{b}\left\|\mathrm{p}-\mathrm{s}_{\mathrm{n}}\right\|\right]+\epsilon_{\mathrm{n}}$ $=\left(1-\alpha_{n}\right)\left\|y_{n}-p\right\|+\alpha_{n} b\left(\left\|\left(1-\beta_{n}\right)\left(p-y_{n}\right)+\beta_{n}\left(p-T y_{n}\right)\right\|+\epsilon_{n}\right.$ $\leq\left(1-\alpha_{n}\right)\left\|y_{n}-p\right\|+\alpha_{n} b\left(1-\beta_{n}\right)\left\|\left(p-y_{n}\right)+\beta_{n}\left(p-T y_{n}\right)\right\|+\epsilon_{n}$ $=\left(1-\alpha_{n}+\alpha_{n} b-\alpha_{n} \beta_{n} b\right)\left\|y_{n}-p\right\|+\alpha_{n} \beta_{n} b\left\|T p-T y_{n}\right\|+\epsilon_{n}$ $=\left(1-\alpha_{n}+\alpha_{n} b-\alpha_{n} \beta_{n} b\right)\left\|y_{n}-p\right\|+\alpha_{n} \beta_{n} b[\varphi(\|p-T p\|)$ $+\mathrm{b}\left\|\mathrm{p}-\mathrm{y}_{\mathrm{n}}\right\|+\epsilon_{\mathrm{n}}$ $=\left(1-\alpha_{n}+\alpha_{n} b-\alpha_{n} \beta_{n} b+\alpha_{n} \beta_{n} b^{2}\right)\left\|y_{n}-p\right\|+\epsilon_{n}$ $\leq\left(1-\alpha_{\mathrm{n}}+\alpha_{\mathrm{n}} \mathrm{b}\right)\left\|\mathrm{y}_{\mathrm{n}}-\mathrm{p}\right\|+\epsilon_{\mathrm{n}}$

Since $0 \leq\left(1-\alpha_{n}+\alpha_{n} b\right)<1$, then using Lemma 1 in (9) yields $\lim _{n \rightarrow \infty}\left\|\mathrm{y}_{\mathrm{n}} \mathrm{p}\right\|=0$. This implies that $\lim _{n \rightarrow \infty} \mathrm{y}_{\mathrm{n}}=\mathrm{p}$.
Conversely, let $\lim _{n \rightarrow \infty} \mathrm{y}_{\mathrm{n}}=\mathrm{p}$. Then,

$\epsilon_{\mathrm{n}}=\left\|\mathrm{y}_{\mathrm{n}+1}-\left(1-\alpha_{\mathrm{n}}\right) \mathrm{y}_{\mathrm{n}}-\alpha_{\mathrm{n}} \mathrm{Ts}_{\mathrm{n}}\right\|$

$\leq\left\|y_{n+1}-p\right\|+\left\|p-\left(1-\alpha_{n}\right) y_{n}-\alpha_{n} T_{n}\right\|$

$=\left\|y_{n+1}-p\right\|+\left\|\left[\left(1-\alpha_{n}\right)+\alpha_{n}\right] p-\left(1-\alpha_{n}\right) y_{n}-\alpha_{n} T_{n}\right\|$

$=\left\|y_{n+1}-p\right\|+\|\left(1-\alpha_{n}\right)\left(p-y_{n}\right)+\alpha_{n}\left(p-\operatorname{Ts}_{n} \|\right.$

$\leq\left\|y_{n+1}-p\right\|+\left(1-\alpha_{n}\right)\left\|y_{n}-p\right\|+\alpha_{n}\left\|p-T s_{n}\right\|$

$=\left\|y_{n+1}-p\right\|+\left(1-\alpha_{n}\right)\left\|y_{n}-p\right\|+\alpha_{n}\left\|\operatorname{Tp}-\operatorname{Ts}_{n}\right\|$

$\leq\left\|y_{n+1}-p\right\|+\left(1-\alpha_{n}\right)\left\|y_{n}-p\right\|+\alpha_{n}[\varphi(\|p-T p\|)$

$\left.+\mathrm{b}\left\|\mathrm{p}-\mathrm{s}_{\mathrm{n}}\right\|\right]$

$=\left\|y_{n+1}-p\right\|+\left(1-\alpha_{n}\right)\left\|y_{n}-p\right\|+\alpha_{n} b \|\left(1-\beta_{n}\right)\left(p-y_{n}\right)$

$+\beta_{\mathrm{n}}\left(\mathrm{p}-\mathrm{T} \mathrm{y}_{\mathrm{n}}\right) \|$

$\leq\left\|y_{n+1}-p\right\|+\left(1-\alpha_{n}\right)\left\|y_{n}-p\right\|+\alpha_{n} b \|\left(1-\beta_{n}\right)\left(p-y_{n}\right)$

$+\alpha_{n} \beta_{n} b\left\|p-T y_{n}\right\|$

$=\left\|y_{n+1}-p\right\|+\left(1-\alpha_{n}+\alpha_{n} b-\alpha_{n} \beta_{n} b\left\|y_{n}-p\right\|\right.$

$\left.+\alpha_{\mathrm{n}} \beta_{\mathrm{n}} \mathrm{b}\right)\|\mathrm{Tp}-\mathrm{Ty}\|$

$\leq\left\|y_{n+1}-p\right\|+\left(1-\alpha_{n}+\alpha_{n} b-\alpha_{n} \beta_{n} b\left\|y_{n}-p\right\|\right.$

$+\alpha_{\mathrm{n}} \beta_{\mathrm{n}} \mathrm{b}\left[\varphi(\|\mathrm{p}-\mathrm{Tp}\|)+\mathrm{b}\left\|\mathrm{p}-\mathrm{y}_{\mathrm{n}}\right\|\right]$

$=\left(1-\alpha_{n}+\alpha_{n} b-\alpha_{n} \beta_{n} b+\alpha_{n} \beta_{n} b^{2}\right)\left\|y_{n}-p\right\|+\epsilon_{n}$

$\leq\left\|\mathrm{y}_{\mathrm{n}+1}-\mathrm{p}\right\|+\left(1-\alpha_{\mathrm{n}}+\alpha_{\mathrm{n}} \mathrm{b}\right)\left\|\mathrm{y}_{\mathrm{n}}-\mathrm{p}\right\| \rightarrow 0$, as $\mathrm{n} \rightarrow \infty$.

This completes the proof.

Remark 3: Theorem 3 in this study is a generalization of Theorem 2 of Osilike ${ }^{[4]}$ which is itself a generalization of Theorem 30 of Rhoades ${ }^{[2]}$.

\section{REFERENCES}

1. Berinde, V., 2002. On the stability of some fixed point procedures, Bul. Stiint. Univ. Baia Mare, Ser. B, Matematica -Informatica, 18: 7-14.

2. Rhoades, B.E., 1991. Some fixed point iteration procedures. Intl. J. Math. Math. Sci., 14: 1-16.

3. Harder, A.M. and T.L. Hicks, 1988. Stability results for fixed point iteration procedures. Math. Japonica, 33: 693-706.

4. Osilike, M.O., 1995. Some stability results for fixed point iteration procedures. J. Nigerian Math. Soc., 14/15: 17-29.

5. Osilike, M.O., 1999. Short proofs of stability results for fixed point iteration procedures for a class of contractive-type mappings. Indian J. Pure Appl. Math., 30: 1229-1234.

6. Rhoades, B.E., 1990. Fixed point theorems and stability results for fixed point iteration procedures. Indian J. Pure Appl. Math. 21: 1-9.

7. Imoru, C.O. and M.O. Olatinwo, 2003. On the stability of Picard and Mann iteration processes. Carpathian J. Math., 19: 155-160. 